\title{
Softening upon Adsorption in Microporous Materials: A Counterintuitive Mechanical Response
}

\author{
Félix Mouhat, ${ }^{\dagger}$ David Bousquet, ${ }^{\ddagger}$ Anne Boutin, ${ }^{\dagger}$ Lila Bouëssel du Bourg, ${ }^{\dagger}$ François-Xavier Coudert, ${ }^{*}{ }^{\dagger}$ \\ and Alain H. Fuchs ${ }^{*}, \dagger$ \\ ${ }^{\dagger}$ PSL Research University, Chimie ParisTech - CNRS, Institut de Recherche de Chimie Paris, 75005 Paris, France \\ *École Normale Supérieure, PSL Research University, Département de Chimie, Sorbonne Universités - UPMC Univ Paris 06, \\ CNRS, 75005 Paris, France
}

\section{Supporting Information}

ABSTRACT: We demonstrate here that microporous materials can exhibit softening upon adsorption of guest molecules, at low to intermediate pore loading, in parallel to the pore shrinking that is well-known in this regime. This novel and counterintuitive mechanical response was observed through molecular simulations of both model pore systems (such as slit pore) and real metal-organic frameworks. It is contrary to common belief that adsorption of guest molecules necessarily leads to stiffening due to increased density, a fact that we show is the high-loading limit of a more complex behavior: a nonmonotonic softeningthen-stiffening.

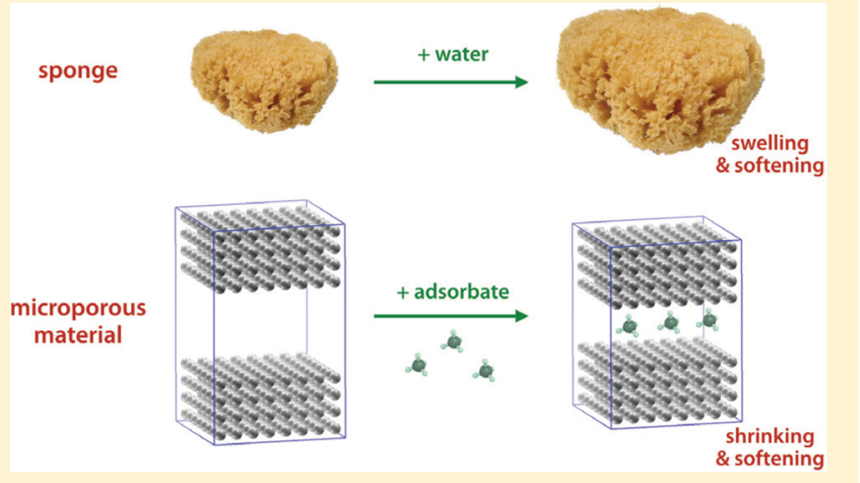

$\mathrm{F}$ luid adsorption in nanoporous materials ${ }^{1-3}$ such as activated carbons, carbon nanotubes, or zeolites has been the subject of numerous studies in the past two decades, owing to its practical importance in such processes as separation, ion exchange, catalysis, biosensing, and controlled drug delivery. For a long time these materials were considered as sufficiently stiff so that their intrinsic flexibility needed not be taken into account in the models employed to understand and predict equilibrium adsorption properties. ${ }^{4-6}$ Indeed the magnitude of the adsorption-induced strain in zeolites is usually of the order of $10^{-3}$ to $10^{-4}$. It was only when transport properties were considered that flexible materials models were tested ${ }^{7,8}$ in order to ascertain whether it could help in better reproducing and explaining the observed experimental trends. Nonetheless, during these years, an important amount of data were collected by the Russian adsorption school of Dubinin and co-workers on the adsorption-induced deformation of microporous carbons and zeolites. These studies were nicely summarized in 2006 in a book by Tvardovskiy. ${ }^{9}$

These features were at least partly overlooked until the issue of adsorption-deformation in mesoporous systems such as MCM-41, SBA-15, or controlled pore glasses (CPGs) was addressed through the study of the phase behavior of confined fluids, with a specific focus on the capillary condensation phenomenon. ${ }^{10}$ In such mesoporous materials, a continuous swelling is observed upon fluid adsorption, up to capillary condensation, and this can be understood in terms of the socalled Bangham effect, ${ }^{11}$ which qualitatively relies on interfacial energy change upon internal surface adsorption. Models have been developed to rationalize this effect. ${ }^{12,13}$
In the case of microporous materials, however, the vapor adsorption phenomenon displays a trend different from what happens in mesoporous materials. At low vapor pressure, the system undergoes a structural contraction followed by swelling at higher vapor pressure. ${ }^{9}$ This peculiar nonmonotonic behavior has been observed experimentally and documented dating back to the $1940 \mathrm{~s},{ }^{14}$ and was recently reported very clearly by Reichenauer and co-workers ${ }^{15}$ in a series of detailed in situ dilatometry experiments of various gases adsorption in synthetic microporous carbons. This nonmonotonic deformation is generic to microporous materials and does not depend on the specifics of host-guest interactions. It contradicts the common intuition that gas adsorption in a confined system increases internal pressures and leads to a continuous volumetric expansion as long as the host framework is flexible (the "sponge" or "balloon picture"). ${ }^{16,17}$

On the theoretical side, Neimark and co-workers developed a nonlocal density functional theory ${ }^{18,19}$ that was able to reproduce this contraction-expansion behavior of adsorption strain in zeolites. Density functional theory and Grand Canonical Monte Carlo simulations were also applied to study adsorption deformation in micropores of activated carbons. ${ }^{20}$ The concept of adsorption stress enabled rationalization of this phenomenon. ${ }^{18,21,22}$ At low gas loading, contraction of the material is driven by the host-guest

Received: September 6, 2015

Accepted: October 11, 2015

Published: October 12, 2015 
dispersion attractive interactions, leading to a negative adsorption stress. At higher loading, once the adsorbed phase becomes denser, short-range repulsive interactions come into play, which lead to a reversal of the adsorption stress, and the material expands in order to accommodate additional guest molecules.

This was a first step toward a comprehensive understanding of the adsorption-deformation coupling phenomena in microporous materials. The next issue to be dealt with is the mechanical stability of the microporous frameworks in the presence of adsorbate species.

Pressure-induced amorphization (PIA) was observed in several porous clathrasils and zeolites ${ }^{23-25}$ as well as in metal-organic frameworks (MOFs) of the zeolitic imidazolate framework (ZIF) family. ${ }^{26,27}$ This structural transition was attributed to the framework mechanical instability due to softening of low-energy phonon modes. ${ }^{28}$ We note in passing that such a mechanism was also proposed for the PIA process in nonporous $\alpha$-quartz ${ }^{29}$ and ice $I_{\mathrm{h}}{ }^{30}$ More recently, PIA in ZIF-8 and some other porous ZIFs was attributed to the framework mechanical instability due to shear mode softening. ${ }^{31,32}$

The phenomenon of pressure-induced amorphization is quite sensitive to the presence of molecules inside the pore space. Insertion of guest molecules was shown to either shift amorphization to higher pressure than in the evacuated material, $^{23,25,31}$ or even to suppress ("deactivate") PIA as in the case of silicalite- $1 .{ }^{33}$ It was then deduced that the presence of adsorbate molecules enhances the stability of the microporous frameworks. This was later supported by a Monte Carlo simulation of silicalite-1, which showed an increase of the computed bulk modulus upon $\mathrm{CO}_{2}$ adsorption. ${ }^{34}$ It should be mentioned however that there are some scarce indications in the literature of opposite behaviors. For instance, Canepa et $\mathrm{al}^{35}$ studied by ab initio simulations the response of MOF-74$\mathrm{Zn}$ when loaded with several molecular gases. They observed that "for almost all adsorbates, the molecular adsorption in the MOF pores induces a substantial increase of the elastic constantsi.e., the MOF looses some of its flexibility". A close examination of their data (Table 3 of ref 35), shows that, while the bulk modulus increases with the insertion of gas molecules, the individual $C_{12}$ and $C_{14}$ elastic constants as well as two of the Young's modulus components decrease when the MOF is loaded with $\mathrm{CO}_{2}$ or $\mathrm{CH}_{4}$ (but not $\mathrm{H}_{2} \mathrm{O}$ ). In many of the cases studied experimentally, the precise value of the gas loading is not well-known, and we presume that many of the experiments were performed at high, if not full, loading. Given that the PIA mechanism involves a collapse of the framework structure around the empty pores, ${ }^{32}$ it seems clear that the mechanical properties can only be enhanced when the pore voids are fully filled with adsorbate molecules. This might not be the case, however, at low gas loading. There is thus a great need to better understand the mechanical response of nanoporous materials upon adsorption, and in particular of soft framework materials such as flexible and stimuli-responsive MOFs. ${ }^{36}$

In this work we examine the elastic response of two model microporous frameworks upon gas adsorption, with a special focus on the initial stage of the adsorption process, i.e., at low gas loading, in order to try understanding the effect of the nonmonotonic volumetric behavior upon adsorption described above on the simultaneous change in the framework mechanical stability.
We performed Monte Carlo (MC) simulations of a LennardJones fluid adsorption in pores of two different geometries: a slit pore, which mimics a MOF framework such as ELM-11, ${ }^{37}$ and a lozenge-shaped pore, which is a simplified model of the MIL-53 family of "breathing" materials ${ }^{38-40}$ (Figure 1). In both

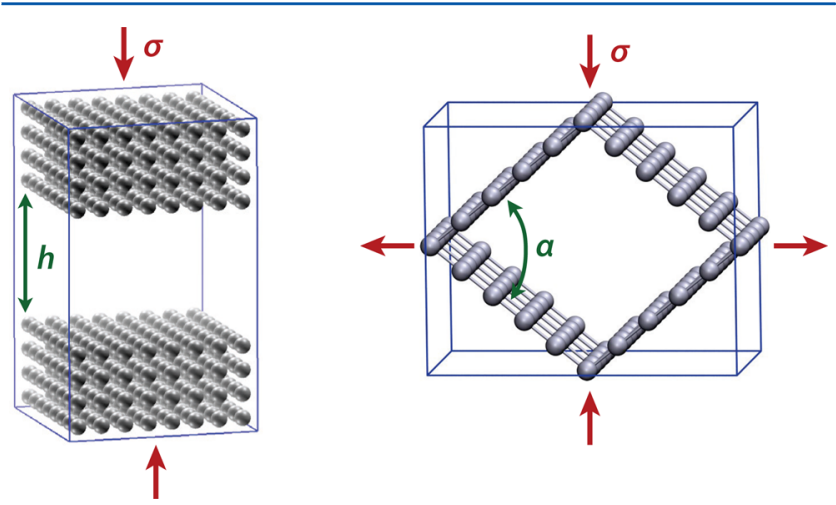

Figure 1. Sketch of the two model microporous frameworks. Left: slit pore; right: lozenge-shaped pore. The red arrows represent the direction of the stress exerted on the solid frameworks, and the unique order parameters of the deformation are represented in green.

cases, the walls of the framework were made of regularly spaced Lennard-Jones particles (more details on the unit cell setup and the force field parameters are given in the Supporting Information). These are very simple model systems for flexible nanoporous materials. They exhibit only one order parameter describing the framework deformation, namely, the width of the slit pore $(h)$ and the opening angle of the lozenge-shaped pore $(\alpha)$; all other dimensions are kept constant. It enables the performance of accurate direct Monte Carlo simulations in the osmotic statistical ensemble $\left(N_{\text {host }} \mu_{\text {ads }}, \sigma, T\right)$, where $N_{\text {host }}$ is the number of host unit cells, $\mu_{\mathrm{ads}}$ is the adsorbate chemical potential, $T$ is the temperature, and $\sigma$ is the mechanical stress (in our case a uniaxial stress) exerted on the system boundaries. It is the appropriate thermodynamic ensemble to treat adsorption in flexible open porous materials, ${ }^{41}$ similar to the Grand isostress ensemble used to describe surface force apparatus (SFA) experiments. ${ }^{42,43}$ For each system, simulations of adsorption were performed at increasing values of guest chemical potential $\mu_{\mathrm{ads}}$, and from each osmotic simulation, the following quantities were extracted: the loading, or average number of adsorbed particles; the deformation of the unit cell, quantified by the average value of $h$ or $\alpha$, and which is directly related to the strain; and the elastic modulus (or stiffness constant) along the deformation mode, calculated from the fluctuations of the unit cell (in Voigt notation, $C_{33}$ for the slit pore, and $C_{66}$ for the lozenge-shaped pore; see Supporting Information for details).

We first performed a series of simulations in the case of the slit pore. The width of the empty pore $h_{0}$ was fixed to the value of $15 \AA$, which corresponds to a typical microporous pore. This width should not be too small in order to avoid spurious effects due to layering that is known to take place in this particular geometry in very narrow pores. ${ }^{42,43}$ We allow the pore width to fluctuate in the osmotic MC simulations, and the free energy of the empty host is simply written in the elastic regime as

$$
F_{\text {host }}(h)=\frac{1}{2} k\left(h-h_{0}\right)^{2}
$$


The harmonic constant $k$ is chosen such that the elastic constant of the bare framework $C_{33}^{0}$ (the Young's modulus of the host) is equal to $10 \mathrm{GPa}$, a rather typical value for MOFs or zeolite microporous materials. The same approach is followed for the lozenge-shaped pore, whose free energy also follows linear elasticity (the mathematical expression for the free energy can be found in a preceding paper ${ }^{39}$ ).

In Figure 2, we report the evolution of the slit pore width and the elastic constant $C_{33}$ as a function of the number of

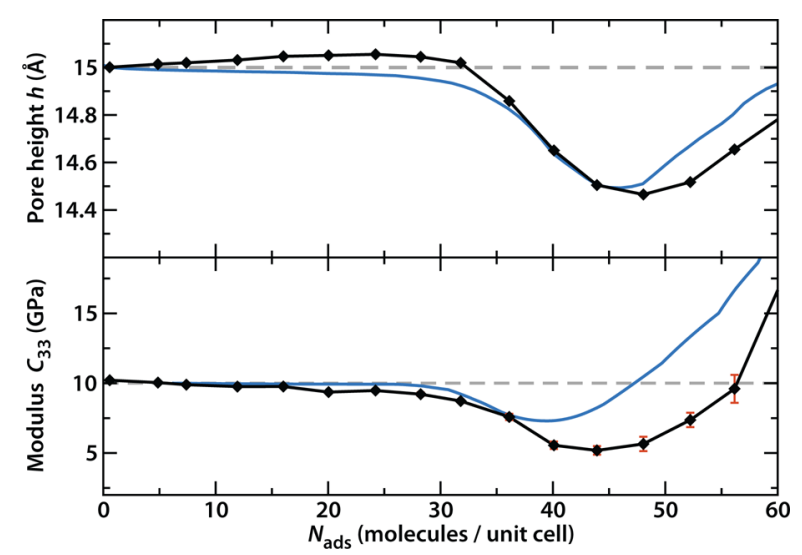

Figure 2. Changes in the slit pore width (upper frame) and in the elastic constant $C_{33}$ (lower frame) as a function of the number of adsorbed molecules. The black curves correspond to the direct osmotic simulations, with uncertainties indicated (when not visible, they are smaller than symbol size). The blue curves correspond to Grand Canonical simulations in which the elastic constant is obtained through the second derivative of $N_{\text {ads }}$ with respect to $h$, using the data of a series of simulations ranging from $h=14$ to $15 \AA$ (details in the Supporting Information). Simulations were performed at $298 \mathrm{~K}$.

adsorbed molecules (pore loading) at $298 \mathrm{~K}$. A slight increase in the pore width at very low $N_{\mathrm{ads}}$ values in the osmotic simulations is observed, which is due to an oscillating solvation pressure effect that is still present, although of small amplitude, in such a $15 \AA$ width slit pore (as well as in additional simulations performed for widths of 18 and $21 \AA$ ). This is in keeping with the fact that this effect is not present in the grand canonical simulations. A rather similar feature was observed by Schoen and co-workers in their simulations of pores of several guest molecular diameters. ${ }^{44}$ This effect is of small amplitude, and strongly dependent on the relative guest size and pore width $h_{0}$.

After that low-pressure effect, the volumetric behavior is nonmonotonic, as expected, and shows a contraction at low to intermediate gas loading followed by an expansion of the pore at high loading. This is the standard contraction-expansion mechanism commonly observed in microporous materials. ${ }^{9,16}$ What is absolutely new and striking is the observation of a nonmonotonic change in the elastic constant upon fluid adsorption. Even in such a simple model as the slit pore, the continuous evolution of elastic stiffness upon adsorption loading has never been reported before. In this particular system, we see that the initial contraction of the porous volume is accompanied by a lowering of the Young's modulus along the $z$ axis, i.e., a softening of the framework. The subsequent expansion at high loading is, on the contrary, accompanied by a stiffening of the framework, as guest molecules fill the micropore. It is worth noting that, on this model whose elastic modulus $(10 \mathrm{GPa})$ is representative of a MOF, the variations in mechanical properties (up to $5 \mathrm{GPa}$ change in $C_{33}$, i.e. a $50 \%$ variation) are quite large, much larger than variations in the pore size itself $(0.6 \AA$ in a pore of $15 \AA)$.

To investigate the influence of geometry, we then studied the evolution of elastic constants upon adsorption in the lozengeshaped pore, from GCMC simulation data at varying pore opening and chemical potential (see Supporting Information). We report in Figure 3 the changes in the order parameter $\alpha$

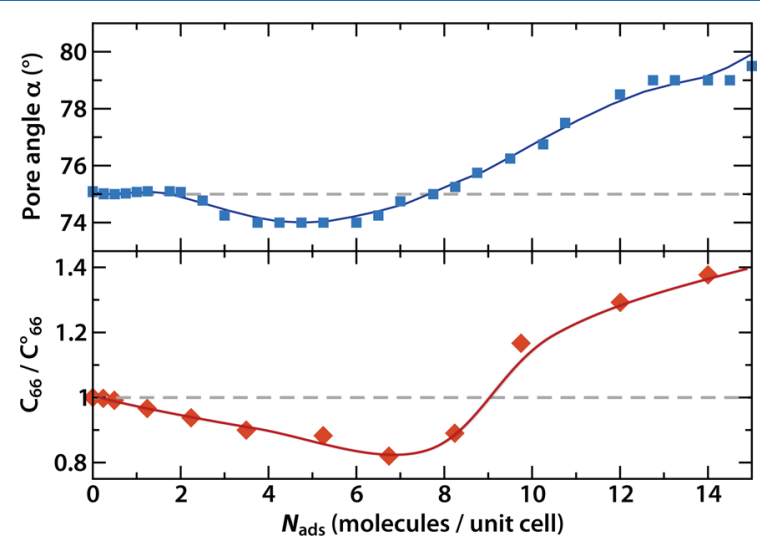

Figure 3. Changes in the lozenge-shaped pore order parameter $\alpha$ (upper frame) and in the elastic constant $C_{66}$ (lower frame) as a function of the number of adsorbed molecules.

(lozenge angle) and the elastic constant $C_{66}$ (shear modulus), as a function of the number of adsorbed molecules at $298 \mathrm{~K}$. Again the nonmonotonic volumetric contraction-expansion behavior is accompanied by a softening-stiffening process upon fluid adsorption. In this particular pore model, adsorptioninduced softening reaches $20 \%$ while the transition from softening to stiffening occurs at lower relative loading. The generic features of adsorption-induced softening-stiffening are, however, strikingly similar to the case of the slit pore.

The question arises now as to whether this behavior of the elastic response of microporous frameworks disclosed here is generic, i.e., whether we can expect to see an adsorptioninduced softening of host-guest systems at partial loading. Obviously more work is needed to be able to fully answer this question; for this, experimental studies at low loading are sorely needed. We have begun, however, to address this question by closely examining the simulation data from our previous molecular dynamics (MD) work on series of MOFs. In the case of cubic material ZIF- $8,{ }^{31}$ no such behavior was observed at low loading in our simulations of methane adsorption. The $\{$ ZIF-8 $\left.+\mathrm{CH}_{4}\right\}$ system exhibits a continuous increase in the computed elastic constants upon methane adsorption. However, another ZIF material, which we have previously studied in order to predict the high pressure stability of ZIF frameworks, ${ }^{32}$ was observed softening upon $\mathrm{CH}_{4}$ adsorption. The $\mathrm{Zn}$ (imidazolate) $)_{2}$ framework of nog topology ${ }^{45}$ exhibits the same nonmonotonic softening-stiffening behavior as our two model pore systems, upon methane adsorption at $300 \mathrm{~K}$. For this material, we show in Figure 4 the evolution of the softest elastic deformation mode, $\lambda_{\min }$, as a function of methane loading. It exhibits a softening by a factor of 2 at low loading, with the modulus going down from 2 to $1 \mathrm{GPa}$ at 20 molecules per unit cell.

We conclude from this first systematic study of mechanical properties of microporous materials under adsorption that a non trivial softening of the microporous \{guest + host \} system 


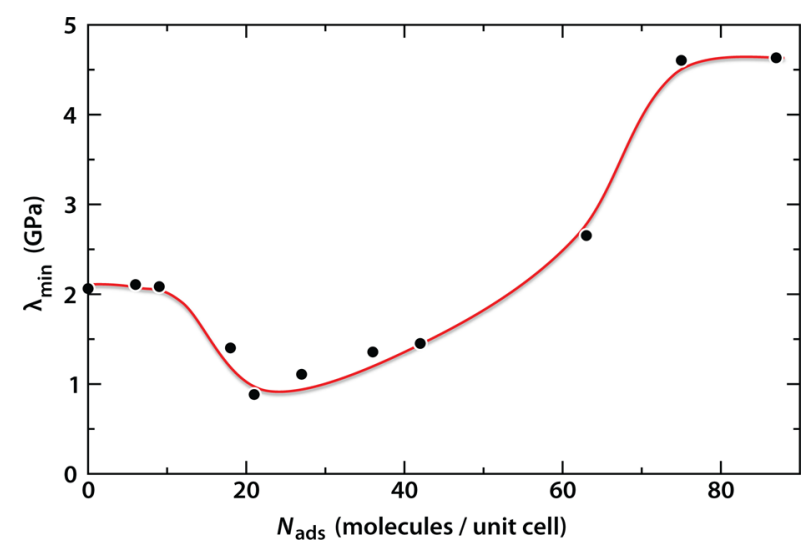

Figure 4. Change in the elastic response of the ZIF $\operatorname{nog}^{45}$ as a function of the number of methane molecules adsorbed species, obtained from isostress-isothermal MD simulations at $300 \mathrm{~K}^{32}$ The ordinate is the smallest elastic modulus of the material, i.e., the lowest eigenvalue $\lambda_{\min }$ of the elastic stiffness tensor. Uncertainty on each value is of the order of $0.1 \mathrm{GPa}$ (see ref 32).

can be observed at low loading, paralleling the contraction of the system. This counterintuitive effect is followed, at higher fluid loading, by the traditionally expected materials stiffening. This phenomenon is rather generic and does not stem from specific interactions, as we have seen it occurring for methane adsorption in pores of various geometries (slit pore, diamondshaped pore, and nog ZIF). However, some microporous frameworks such as ZIF-8 do not show such a behavior for reasons that are not yet clear. It is possible that only some of the components of the framework elastic response experience such a temporary softening in the low loading regime. Work on a larger number of host-guest systems is in progress to clear up this point.

This nonmonotic softening-stiffening evolution of mechanical properties, proposed on the basis of molecular simulations, can explain some results from the earlier literature. In particular, it explains the MOF softening upon adsorption found (but not commented upon) by Canepa et al. in their ab initio studies of $\mathrm{CO}_{2}$ and $\mathrm{CH}_{4}$ adsorption in MOF-74-Zn. ${ }^{35}$ It can also help explain the recent counterintuitive experimental results of Alabarse et al., who showed that the presence of water within the pores of $\mathrm{AlPO}_{4}-54$ lead to a decrease in mechanical stability, attested by the occurrence of pressure-induced amorphization at lower pressure than in the guest-free $\mathrm{AlPO}_{4}-54{ }^{46}$ This last example shows the importance of better understanding the influence of guest adsorption on mechanical properties of microporous materials, as it can have a drastic impact not only on the modification of the elastic behavior (thus augmenting, through softening, the magnitude of adsorption deformation) but also on the stability under pressure, a key property for many industrial applications.

\section{ASSOCIATED CONTENT}

\section{S Supporting Information}

The Supporting Information is available free of charge on the ACS Publications website at DOI: 10.1021/acs.jpclett.5b01965.

Full description of the pore models, molecular simulation procedures, and calculation of elastic constants (PDF) Complete set of input files, corresponding to nine $\mathrm{CH}_{4}$ molecules in ZIF nog (as well as the NAMD 2.9 software patch) (ZIP)

\section{AUTHOR INFORMATION}

\section{Corresponding Authors}

*E-mail: alain.fuchs@cnrs-dir.fr.

*E-mail: fx.coudert@chimie-paristech.fr. Twitter: @fxcoudert.

\section{Notes}

The authors declare no competing financial interest.

\section{ACKNOWLEDGMENTS}

We thank Alex Neimark and Gudrun Reichenauer for fruitful discussions. We acknowledge funding from Agence Nationale de la Recherche (project ANR-2010-BLAN-0822) and PSL Research University (project MECADS, grant ANR-10-IDEX0001-02), and computing time on HPC platforms by GENCI (Grant x2015087069).

\section{REFERENCES}

(1) Porous materials in which the pore diameters are $<2 \mathrm{~nm}$ are called "microporous", following IUPAC recommendations. ${ }^{3}$ Materials that exhibit pore diameters larger than $2 \mathrm{~nm}$ are called "mesoporous". The present study is mostly devoted to understanding the effect of adsorption on compliant microporous materials.

(2) Rouquerol, F.; Rouquerol, J.; Sing, K. Adsorption by Powders and Porous Solids; Academic Press: San Diego, CA, 1999.

(3) Rouquerol, J.; Avnir, D.; Fairbridge, C. W.; Everett, D. H.; Haynes, J. M.; Pernicone, N.; Ramsay, J. D. F.; Sing, K. S. W.; Unger, K. K. Pure Appl. Chem. 1994, 66, 1739-1758.

(4) Cracknell, R. F.; Gubbins, K. E.; Maddox, M.; Nicholson, D. Acc. Chem. Res. 1995, 28, 281.

(5) Smit, B. Phys. Scr. 1996, 66, 80.

(6) Fuchs, A. H.; Cheetham, A. K. J. Phys. Chem. B 2001, 105, 7375.

(7) Auerbach, S. M. Int. Rev. Phys. Chem. 2000, 19, 155.

(8) Leroy, F.; Rousseau, B.; Fuchs, A. H. Phys. Chem. Chem. Phys. 2004, 6, 775.

(9) Tvardovskiy, A. V. Sorption Deformation; Elsevier: Amsterdam, 2006.

(10) Zickler, G. A.; Jähnert, S.; Wagermaier, W.; Funari, S. S.; Findenegg, G. H.; Paris, O. Phys. Rev. B: Condens. Matter Mater. Phys. 2006, 73, 184109.

(11) Bangham, D. H.; Fakhoury, N. Nature 1928, 122, 681.

(12) Ravikovitch, P. I.; Neimark, A. V. Langmuir 2006, 22, 11171.

(13) Gor, G. Y.; Neimark, A. V. Langmuir 2010, 26, 13021.

(14) Haines, R. S.; McIntosh, R. J. Chem. Phys. 1947, 15, 28-38.

(15) Baltzer, C.; Reichenauer, G.; Wiener, M. Poromechanics V: Proceedings of the Fifth Biot Conference on Poromechanics; American Society of Civil Engineers: Reston, VA, 2013; p 701.

(16) Coudert, F.-X.; Fuchs, A. H.; Neimark, A. V. Phys. Chem. Chem. Phys. 2014, 16, 4394.

(17) Joo, J.; Kim, H.; Han, S. S. Phys. Chem. Chem. Phys. 2013, 15, $18822-18826$.

(18) Ravikovitch, P. I.; Neimark, A. V. Langmuir 2006, 22, 10864.

(19) Kowalczyk, P.; Ciach, A.; Neimark, A. V. Langmuir 2008, 24, 6603.

(20) Do, D. D.; Nicholson, D.; Do, H. D. J. Phys. Chem. C 2008, 112, 14075 .

(21) Neimark, A. V.; Coudert, F.-X.; Boutin, A.; Fuchs, A. H. J. Phys. Chem. Lett. 2010, 1, 445.

(22) Coudert, F.-X.; Boutin, A.; Fuchs, A. H.; Neimark, A. V. J. Phys. Chem. Lett. 2013, 4, 3198.

(23) Tse, J. S.; Klug, D. D.; Ripmeester, J. A.; Desgreniers, S.; Lagarec, K. Nature 1994, 369, 724-727.

(24) Gillet, P.; Malezieux, J.-M.; Itie, J.-P. Am. Mineral. 1996, 81, 651-657.

(25) Huang, Y.; Havenga, E. A. Chem. Phys. Lett. 2001, 345, 65-71.

(26) Chapman, K. W.; Halder, G. J.; Chupas, P. J. J. Am. Chem. Soc. 2009, 131, 17546-17547. 
(27) Bennett, T. D.; Simoncic, P.; Moggach, S. A.; Gozzo, F.; Macchi, P.; Keen, D. A.; Tan, J.-C.; Cheetham, A. K. Chem. Commun. 2011, 47, $7983-7985$.

(28) Greaves, G. N.; Meneau, F.; Majerus, O.; Jones, D. G.; Taylor, J. Science 2005, 308, 1299-1302.

(29) Tse, J. S.; Klug, D. D. Phys. Rev. Lett. 1991, 67, 3559-3562.

(30) Tse, J. S. J. Chem. Phys. 1992, 96, 5482-5487.

(31) Ortiz, A. U.; Boutin, A.; Fuchs, A. H.; Coudert, F.-X. J. Phys. Chem. Lett. 2013, 4, 1861.

(32) Bouëssel du Bourg, L.; Ortiz, A. U.; Boutin, A.; Coudert, F.-X. APL Mater. 2014, 2, 124110.

(33) Haines, J.; Cambon, O.; Levelut, C.; Santoro, M.; Gorelli, F.; Garbarino, G. J. Am. Chem. Soc. 2010, 132, 8860-8861.

(34) Coasne, B.; Haines, J.; Levelut, C.; Cambon, O.; Santoro, M.; Gorelli, F.; Garbarino, G. Phys. Chem. Chem. Phys. 2011, 13, 2009620099.

(35) Canepa, P.; Tan, K.; Du, Y.; Lu, H.; Chabal, Y. J.; Thonhauser, T. J. Mater. Chem. A 2015, 3, 986-995.

(36) Coudert, F.-X. Chem. Mater. 2015, 27, 1905-1916.

(37) Numaguchi, R.; Tanaka, H.; Watanabe, S.; Miyahara, M. T. J. Chem. Phys. 2014, 140, 044707.

(38) Férey, G.; Serre, C. Chem. Soc. Rev. 2009, 38, 1380.

(39) Bousquet, D.; Coudert, F.-X.; Fossati, A. G. J.; Neimark, A. V.; Fuchs, A. H.; Boutin, A. J. Chem. Phys. 2013, 138, 174706.

(40) Bousquet, D.; Coudert, F.-X.; Boutin, A. J. Chem. Phys. 2012, 137, 044118.

(41) Coudert, F.-X.; Jeffroy, M.; Fuchs, A. H.; Boutin, A.; MellotDraznieks, C. J. Am. Chem. Soc. 2008, 130, 14294.

(42) Bordarier, P.; Rousseau, B.; Fuchs, A. H. J. Chem. Phys. 1997, 106,7295 .

(43) Bordarier, P.; Schoen, M.; Fuchs, A. H. Phys. Rev. E: Stat. Phys., Plasmas, Fluids, Relat. Interdiscip. Top. 1998, 57, 1621.

(44) Günther, G.; Prass, J.; Paris, O.; Schoen, M. Phys. Rev. Lett. 2008, 101, 086104.

(45) Tian, Y.-Q.; Zhao, Y.-M.; Chen, Z.-X.; Zhang, G.-N.; Weng, L.H.; Zhao, D.-Y. Chem. - Eur. J. 2007, 13, 4146.

(46) Alabarse, F. G.; Silly, G.; Haidoux, A.; Levelut, C.; Bourgogne, D.; Flank, A.-M.; Lagarde, P.; Pereira, A. S.; Bantignies, J.-L.; Cambon, O.; Haines, J. J. Phys. Chem. C 2014, 118, 3651-3663. 